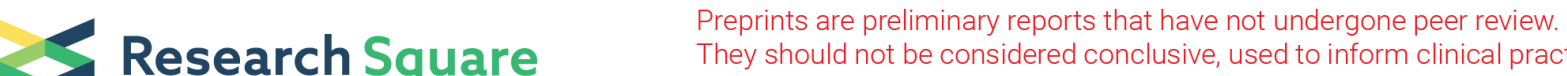 They should not be considered conclusive, used to inform clinical practice, or referenced by the media as validated information.
}

\section{MRI-guided Radiotherapy Identifies Early Pseudoprogression of Glioblastoma}

\section{Kolton K Jones}

University of Miami https://orcid.org/0000-0002-7510-7617

\section{Sarah Dooley \\ University of Miami \\ Danilo Maziero \\ University of Miami \\ John C Ford \\ University of Miami \\ Radka Stoyanova \\ University of Miami \\ Mohammed Goryawala \\ University of Miami}

\section{Tejan Diwanji}

University of Miami

Eric A Mellon ( $\square$ eric.mellon@med.miami.edu )

University of Miami

\section{Research}

Keywords: Glioblastoma, MRI, radiotherapy, MRI-guided radiotherapy, pseudoprogression

Posted Date: July 17th, 2020

DOI: https://doi.org/10.21203/rs.3.rs-42434/v1

License: (c) (1) This work is licensed under a Creative Commons Attribution 4.0 International License.

Read Full License 


\section{Abstract}

Background The standard glioblastoma treatment paradigm consists of surgery, pre-radiotherapy MRI, six weeks of chemoradiotherapy, followed by post-radiotherapy MRI and continued adjuvant temozolomide. In a significant proportion of patients, post-radiotherapy MRI demonstrates tumor enlargement due to either treatment failure (true progression) or treatment response (pseudoprogression). Recently introduced MRI-guided radiotherapy systems obtain daily MRI of glioblastoma patients, and we hypothesized that progression can be identified early during radiotherapy.

Methods Fourteen glioblastoma patients underwent tri-Cobalt-60 MRI-guided radiotherapy in 30 fractions over 6 weeks delivered with concurrent temozolomide. The tumor target volume was delineated on MRI before each of the 30 fractions. The images obtained by the $0.35 \mathrm{~T}$ MRI-guided system is shown to be similar to T2-weighted images obtained by a clinical diagnostic MRI-scanner. Hyperintense volumes were measured over time through radiotherapy.

Results Four of fourteen patients demonstrated increases of at least $25 \%$ and $1.5 \mathrm{cc}$ in T2 hyperintense volume through radiation therapy. This volume expansion correlated with both T2/FLAIR and contrastenhanced volume expansion on post-radiotherapy diagnostic MRIs. In three of four cases, significant volume growth only started at week three of treatment, with the most prominent changes occurring during weeks four and five. While patient numbers are limited, patients with growth during therapy exhibited excellent survival, consistent with the known improved survival of patients with pseudoprogression.

Conclusions Daily MRI acquisition during radiotherapy identifies early pseudoprogression typically starting during week 3 or 4 of treatment. This and other daily MRI techniques during radiotherapy could enable early adaptation of therapy in glioblastoma patients.

\section{Background}

Glioblastoma (GBM) is the most aggressive brain tumor in adults (1). Standard clinical treatment for GBM consists of tumor resection followed by six weeks of radiation therapy (RT) with temozolomide, resulting in a median survival of about 15 months (2). Magnetic Resonance Imaging (MRI) following the initial six week treatment period reveals gadolinium-enhanced volume increase in up to $49 \%$ of patients (3). Of patients with this volume increase between pre and post radiotherapy scans, about one-third result from progressive tumor growth and two-thirds are due to therapy changes termed pseudoprogression (46). True progression is characterized by continuous tumor growth despite treatment with a poor median survival 10 months; while pseudoprogression reflects therapy response with recruitment of blood vessels and/or necrosis and improved median survival $~ 38$ months $(3,7)$. No current clinical modality can reliably separate true and pseudoprogression at early time points. Therefore, patients are typically managed without therapy change for many additional months to determine whether changes are progressive (true progression) or spontaneously improve (pseudoprogression). 
Early differentiation of true and pseudoprogression would enable modification of radiotherapy or chemotherapy and prevent unnecessary surgeries (8). Brain biopsies are invasive, and thus there are strong efforts towards using non-invasive methods such as MRI to predict eventual patient survival based on early changes $(8,9)$. Typically, MRI is obtained approximately two weeks before beginning a six weeks course of chemoradiation and then again four weeks after completion, for a total time interval of three months between scans. Previous studies have assessed different modalities of MRI such as T1weighted (10), T2-weighted (11), perfusion (12) diffusion weighted imaging (DWI) (13), and radiomics (14) at these several month intervals. However, there is limited data analyzing changes that occur during the radiotherapy treatment time interval because it has not been feasible to perform frequent MRI scans due to the cost and logistics of standalone MRI scans. Therefore, the kinetics of change during radiotherapy has not been fully elucidated.

Recently, the combination of MRI and radiotherapy (MRI-RT) devices has become available, providing the opportunity to acquire daily MRI for radiotherapy patients $(15,16)$. We observed that GBM patients treated with MRI-RT at our institution had a variable time course of changes during treatment that we hypothesize might provide additional information for predicting treatment outcome and adapting radiotherapy treatment. Here we provide our preliminary experience with 14 GBM patients treated on the MRI-RT device and describe the visualized changes during radiotherapy.

\section{Methods}

\section{Patients}

Fourteen glioblastoma patients, ten men and four women ages $24-78$, underwent RT delivered by triCobalt-60 MRI-RT with concurrent temozolomide (TMZ) at the University of Miami's Miller School of Medicine Sylvester Comprehensive Cancer Center. All patients provided written consent under an institutional umbrella protocol for MRI-RT to analyze imaging findings and outcomes. Seven patients underwent complete resection, one had a partial resection, and six had biopsy alone. Four patients had IDH-1 R132H mutation by immunohistochemistry and six patients had hypermethylation of the MGMT promoter region. Kaplan-Meier survival plots were generated using SPSS (IBM SPSS Statistics version 26).

\section{Radiation therapy planning and delivery}

Two to four weeks after surgery patients underwent a pre-treatment diagnostic $1.5 \mathrm{~T}$ or $3 \mathrm{~T}$ MRI including T1-weighted after gadolinium contrast and T2-weighted scans. Simulation was performed at that time on the tri-cobalt MRIdian system (ViewRay, Mountain View, USA) in position for treatment including MRI compatible thermoplastic mask (Civco, Coralville, USA). Vendor supplied 6-channel surface array posterior torso coil was placed under the masking system and 5-channel surface array anterior head and neck coil was wrapped around the mask anteriorly. Patients received $60 \mathrm{~Gy}$ in 30 fractions in a single volume with clinical target volume (CTV) expansion of $2 \mathrm{~cm}$ on the cavity and enhancing tumor and 
planning target volume expansion of $0.3 \mathrm{~cm}$ concurrent with temozolomide $75 \mathrm{mg} / \mathrm{mg}^{2}$ using MRI-RT with daily MRI ${ }^{(2)}$.

\section{Imaging acquisition}

The radiation treatment system is equipped with a $0.35 \mathrm{~T} \mathrm{MRI} \mathrm{(Siemens} \mathrm{Avanto,} \mathrm{version} \mathrm{syngo} \mathrm{MR} \mathrm{B17,}$ IDEA version VB19, Siemens Medical Solutions, Erlangen, Germany). The scanner includes a balanced steady-state free precession (bSSFP) pulse sequence (17). The bSSFP is commonly used in relatively low field MRI acquisition due to its capability of providing an increased signal-to-noise ratio with higher temporal resolution $(18,19)$. The signal originated by the bSSFP sequence is also known for its mixed composition that is weighted by T1 and T2. However, the use of high flip angles produces high T2weighting as implemented on this $0.35 \mathrm{~T}$ system (17). The bSSFP-derived images were acquired with the following parameters: $\mathrm{TE}$ (time to echo) $/ \mathrm{TR}$ (time to repetition) $=1.92 \mathrm{~ms} / 3.84 \mathrm{~ms}$, flip angle $=60$, acquisition matrix $=266 \times 288 \times 266$, Voxel size $1.5 \mathrm{~mm} \times 1.5 \mathrm{~mm} \times 1.5 \mathrm{~mm}$ (3D-acquisition), coil elements VAP, VAS, VPP, VPS, bandwidth $532 \mathrm{~Hz} / \mathrm{Px}$, Gradient mode: fast and acquisition time $=2$ minutes and 8 seconds. This choice of acquisition parameters provided whole head images with contrast comparable to the obtained from T2-fast spin-echo images acquired from a diagnostic 1.5 T scanner (Signa HDxt, GE Healthcare, Chicago, USA), as illustrated in Fig. 1.

\section{Image analysis}

The volume of hyperintensity on the bSSFP images from each treatment fraction was contoured manually using MIM (version 6.7, Cleveland, USA). Tumors were reported as a significant change if the volume changed at least 25\% (similar to Macdonald's criteria for progressive disease (20)) between any two time points (e.g. fraction 1 compared to fraction 30 ) and that change was greater than $1.5 \mathrm{cc}$ as a threshold to ensure no changes were simply due to contouring variability.

Patients received post-treatment MRI 3-6 weeks after completion of concurrent chemoradiotherapy and continued adjuvant chemotherapy. Patients with observed T2-weighted growth on this study were all noted by post-treatment radiology report to have growth of enhancement and edema on post-treatment MRI.

\section{Results}

Four out of fourteen patients (1, 2, 3 and 8) demonstrated an increase of at least $25 \%$ and $1.5 \mathrm{cc}$ in hyperintense volume during radiation therapy. All of these patients were confirmed to have possible pseudoprogession or true progression by inspection and radiology report on post-treatment postgadolinium MRI.

Patient 1 (Fig. 2), a 65 year old man, presented to radiation oncology with multifocal glioblastoma (IDH-1 mutation negative, MGMT nonhypermethylated) in the right temporal lobe after biopsy of a discrete lesion about $1 \mathrm{~cm}$ inferior to the axial slice shown in Fig. 2A. A tiny $(\sim 2 \mathrm{~mm})$ area of equivocal T2 change 
was observed as shown, and this area was included in the 60 Gy CTV. Little change was observed in this area until about fraction 18 (late week 4), at which time rapid growth was seen until fraction 26 (week 6) when the size plateaued. No steroids were used at any time during the radiotherapy treatment. The observed findings during treatment were consistent with one-month post treatment diagnostic imaging (Fig. 2A, bottom two panels). Bevacizumab was added to temozolomide after the diagnostic MRI with dramatic improvement in MRI findings. The patient died of multi-focal glioblastoma, mostly out of the initial radiation therapy field, 24 months after biopsy.

Patient 2 (Fig. 3), a 47 year old man, underwent gross total resection of a right temporal glioblastoma (IDH-1 mutation negative, MGMT nonhypermethylated). On treatment planning MRI two weeks later a tiny area of enhancement without surrounding edema was observed in the right frontal lobe suspicious for multifocal disease and added to the 60 Gy CTV. Post-operative dexamethasone taper was stopped at fraction 1 and no further steroids were given during treatment. Similar to the pre-treatment T2 images, no change in the multi-focal area was visualized on the MRI-RT scans initially. Growth was identified on MRIRT early in week 3 which accelerated through week 4 and 5 and plateaued in week 6 . This area continued to progress and underwent resection about 6 months after completion of radiation. Pathology demonstrated only reactive changes and inflammation with no clear tumor. The patient went to hospice 20 months after initial resection due to additional foci of progression outside of the radiation therapy field.

Patient 8 (Fig. 4), a 59 year old man, underwent gross total resection of a right parietal glioblastoma (IDH1 mutation negative, MGMT nonhypermethylated). Patient was tapered from dexamethasone $4 \mathrm{mg}$ twice daily and maintained at $2 \mathrm{mg}$ twice daily after the start of treatment due to left hand weakness.

Significant growth of T2 hyperintensity was identified starting fraction 17 and progressing nearly linearly through fraction 30 . Temozolomide chemotherapy was continued through currently 9 months of followup.

Among the four patients with $25 \%$ or more growth during treatment, we observed non-linear growth kinetics (Fig. 5). Specifically, three of the patients (numbers 1, 2, and 8) demonstrated little change during weeks $1-3$ of radiotherapy followed by significant growth starting in week 4 . Patient 3 had growth in the first week that plateaued during week 3 . Another observation was that three of the patients (numbers 1,2 , and 3) had a plateau in growth during the last week of treatment or earlier, while patient 8 continued to grow through week 6 . The bSSFP MRIs from the sixth week of treatment for all fourteen patients demonstrated similar T2-FLAIR abnormalities when compared to the patients' diagnostic T2 MRIs posttreatment completion.

Nine of the fourteen patients are alive after finishing the chemoradiation treatment. Median follow-up was 19.1 months (minimum 6 months) with estimated survival of 23.9 months (Fig. 6). A non-significant difference in estimated survival is seen at 18 months $(100 \%$ for patients with growth during treatment vs. $67 \%$ without, $p=0.45$ ), which supports the likely pseudoprogression observed in these patients. 
Regarding patients with more minor changes, one patient (patient 7 shown in Fig. 1) had a 23\% decrease in bSSFP hyperintense volume beginning at fraction $18(35.7 \mathrm{cc})$ trending nearly linearly to fraction 30 (29.0 cc). Patient 5 had a 15\% increase in T2 hyperintense volume almost linearly starting at fraction 12 $(23.5 \mathrm{cc})$ and plateauing after fraction $19(26.8 \mathrm{cc})$. Patients 11 and 13 underwent gross total resections and the resection cavities were observed to shrink from 1.9 to $0.6 \mathrm{cc}$ and 82.6 to $72 \mathrm{cc}$, respectively. All of these changes were also reported on diagnostic radiology report post-treatment. The remaining patients had less than $10 \%$ or $1.5 \mathrm{cc}$ change of hyperintense volume throughout the treatment period.

\section{Discussion}

This is the first analysis of daily MRI changes during radiotherapy of glioblastoma. Four (29\%) of fourteen patients were observed with at least $25 \%$ and $1.5 \mathrm{cc}$ of growth of the T2-weighted volume during radiotherapy, consistent with known rates of progression on MRI comparing pre-treatment MRI and MRIs obtained in the 1 month post-treatment period (21). Only one patient was observed with reduction in volume. In these patients where changes were present, the majority of patients exhibited the bulk of changes late during the treatment course-starting in week 3-4 and becoming most prominent in weeks 5 and 6 . Volume changes correlated with progressive gadolinium enhancement and T2-weighted changes at one month post-treatment. The patients with growth during treatment had a non-significant improvement on the survival curve suggestive of the improved survival observed with pseudoprogression (3).

Prior work has demonstrated that MRI at one or two time points during radiotherapy correlates with overall survival. For example, Galban et al. mapped perfusion changes from baseline at week one and three in 44 patients with GBM and found correlations with overall survival based on these changes (22). Hamstra et al. mapped apparent diffusion coefficient changes from baseline at week one, three, and ten (3-4 weeks post radiotherapy) in 60 patients with GBM and found that changes at week three had the most correlation with survival (23). Nelson et al. obtained magnetic resonance spectroscopic imaging in 25 patients before, during, and one month after radiotherapy and found that decreases in volumes demonstrating elevated choline to $\mathrm{N}$-acetylaspartate ratios mid-treatment correlated with survival (24). This is encouraging that midpoint MRI or spectroscopy may be useful to determine prognosis and potentially adapt treatment. Nevertheless, our results suggest that acquiring MRI early in the treatment course may be sub-optimal for correlations with eventual outcome. For example, Patients 1, 2 and 8 (Fig. 5A, B and D, respectively) only showed T2-weighted volume increase after the fourth week of treatment, indicating that studies analyzing changes during week three or earlier may be premature.

Additionally, there is significant heterogeneity in treatment planning margins used for treatment of glioblastoma (25). While we have planned these cases based on EORTC margins (single $2 \mathrm{~cm}$ CTV expansion from resection cavity and enhancement to $60 \mathrm{~Gy}$ ) (26), the Adult Brian Tumor Consortium (ABTC) uses the typically much smaller margins of $5 \mathrm{~mm}$ CTV expansion from edema to 46 Gy and $5 \mathrm{~mm}$ CTV expansion on cavity and enhancement to $60 \mathrm{~Gy}$ (27). Groups using the ABTC margins have reported no difference in treatment failure rates (28-30), though this is not without controversy (31). 
Interestingly, in our study we found that in three of fourteen patients by the end of week 4 (fraction 20) the edema had expanded at least $5 \mathrm{~mm}$ from its initial extent $(+5 \mathrm{~mm},+1 \mathrm{~cm},+1.1 \mathrm{~cm})$. Although the clinical significance of these findings with regards to treatment margin or dose alterations is unclear, it can now be investigated prospectively since such growth during radiotherapy is now identifiable.

Our study is limited by single pulse sequence acquisition during radiotherapy. Studies are underway at multiple institutions to obtain multiparametric MRI longitudinally on the MRI-RT system to address this question $(32,33)$. In particular, most criteria for growth and progression are at least partly based on gadolinium enhancement (34). We did not give extra gadolinium to patients during radiotherapy in this preliminary study given recent concerns about gadolinium toxicity (35). However, given these findings we do plan to dose gadolinium for 1-2 additional timepoints during RT in future patients for response to therapy and radiotherapy treatment adaptation as well as investigate non-invasive perfusion methods during RT $(36,37)$. Nevertheless, the association of edema during radiotherapy with post-treatment contrast volume increase suggests that the two are related in this study. Another possible confounding is that both enhancement and edema volume can be sensitive to changes in steroid doses (38). Our default is not to use steroids in patients during radiotherapy unless the patient is symptomatic, and most did not receive steroids after the start of treatment. Thus, we have found no clear correlation between steroids and volume as noted in the case descriptions.

\section{Conclusions}

We identified probable pseudoprogression in four of fourteen glioblastomas during MRI-guided primary six week chemoradiotherapy by daily T2-weighted MRI volumes. In three of four cases, significant volume growth was not observed until at least week three of treatment, with the most prominent changes occurring during weeks 4 and 5 . Further studies are underway to identify whether the kinetics of volume growth, changes in multiparametric MRI, or radiomics changes of daily MRI during radiotherapy may correlate with overall survival in an increased number of patients towards early adaptation of therapy in glioblastoma patients.

\section{List Of Abbreviations}

RT, radiation therapy; MRI, magnetic resonance imaging; DWI, diffusion weighted imaging; GBM, glioblastoma; TMZ, temozolomide; IDH-1, isocitrate dehydrogenase 1; MGMT, O[6]-methylguanine-DNA methyltransferase; CTV, clinical target volume; bSSFP, balanced steady state free precession; ABTC, adult brain tumor consortium; EORTC, European Organisation for Research and Treatment of Cancer

\section{Declarations}

Ethical Approval and Consent to participate 
The studies herein were approved by the University of Miami Institutional Review Board 20160817. All patients gave informed consent.

\section{Consent for publication}

Consent for publication is not applicable. Included data is not identifiable to any individual person.

\section{Availability of supporting data}

The datasets used during the current study are available from the corresponding author on reasonable request.

\section{Competing interests}

The authors declare that they have no competing interests.

\section{Funding}

The project described was supported by the National Cancer Institute and National Center For Advancing Translational Sciences of the National Institutes of Health under Award Numbers K12CA226330 and UL1TR002736. The authors would also like to thank the Sylvester Comprehensive Cancer Center for their support of this work. These funders provided salary support for some of the authors and for study coordination, but did not contribute to study design, collection, analysis, or interpretation of data. The content is solely the responsibility of the authors and does not necessarily represent the official views of the National Institutes of Health or Sylvester Comprehensive Cancer Center. Publication fees were supported by Viewray, Inc. Viewray, Inc. did not contribute to study design, collection, analysis, or interpretation of data.

\section{Authors' contributions}

EAM conceived, designed, and substantially revised this study.

$\mathrm{KJ}, \mathrm{SD}$, and DM analyzed the data and co-wrote the manuscript.

JCF and TD substantially contributed to acquisition of the data.

RS, MG, and TD substantially contributed to data analysis and interpretation

\section{Acknowledgements}

We would like to acknowledge our funders in the funding statement above.

\section{References}


1. Ostrom QT, Gittleman H, Farah P, Ondracek A, Chen Y, Wolinsky Y, et al. CBTRUS statistical report: Primary brain and central nervous system tumors diagnosed in the United States in 2006-2010. Neuro Oncol. 2013;15 Suppl 2:ii1-56.

2. Stupp R, Mason WP, van den Bent MJ, Weller M, Fisher B, Taphoorn MJ, et al. Radiotherapy plus concomitant and adjuvant temozolomide for glioblastoma. The New England journal of medicine. 2005;352(10):987-96.

3. Brandes AA, Franceschi E, Tosoni A, Blatt V, Pession A, Tallini G, et al. MGMT Promoter Methylation Status Can Predict the Incidence and Outcome of Pseudoprogression After Concomitant Radiochemotherapy in Newly Diagnosed Glioblastoma Patients. Journal of Clinical Oncology. 2008;26(13):2192-7.

4. Brandes AA, Tosoni A, Spagnolli F, Frezza G, Leonardi M, Calbucci F, et al. Disease progression or pseudoprogression after concomitant radiochemotherapy treatment: pitfalls in neurooncology. Neuro Oncol. 2008;10(3):361-7.

5. Chaskis C, Neyns B, Michotte A, De Ridder M, Everaert H. Pseudoprogression after radiotherapy with concurrent temozolomide for high-grade glioma: clinical observations and working recommendations. Surgical neurology. 2009;72(4):423-8.

6. Fabi A, Russillo M, Metro G, Vidiri A, Di Giovanni S, Cognetti F. Pseudoprogression and MGMT status in glioblastoma patients: implications in clinical practice. Anticancer research. 2009;29(7):2607-10.

7. Young RJ, Gupta A, Shah AD, Graber JJ, Zhang Z, Shi W, et al. Potential utility of conventional MRI signs in diagnosing pseudoprogression in glioblastoma. Neurology. 2011;76(22):1918-24.

8. Juratli TA, Engellandt K, Lautenschlaeger T, Geiger KD, von Kummer R, Cerhova J, et al. Is there pseudoprogression in secondary glioblastomas? Int J Radiat Oncol Biol Phys. 2013;87(5):1094-9.

9. Thust SC, van den Bent MJ, Smits M. Pseudoprogression of brain tumors. Journal of magnetic resonance imaging: JMRI. 2018.

10. Reddy K, Westerly D, Chen C. MRI patterns of T1 enhancing radiation necrosis versus tumour recurrence in high-grade gliomas. Journal of medical imaging and radiation oncology. 2013;57(3):349-55.

11. Booth TC, Larkin TJ, Yuan Y, Kettunen MI, Dawson SN, Scoffings D, et al. Analysis of heterogeneity in T2-weighted MR images can differentiate pseudoprogression from progression in glioblastoma. PloS one. 2017;12(5):e0176528.

12. Sugahara T, Korogi Y, Kochi M, Ikushima I, Hirai T, Okuda T, et al. Correlation of MR imagingdetermined cerebral blood volume maps with histologic and angiographic determination of vascularity of gliomas. AJR American journal of roentgenology. 1998;171(6):1479-86.

13. Reimer C, Deike K, Graf M, Reimer P, Wiestler B, Floca RO, et al. Differentiation of pseudoprogression and real progression in glioblastoma using $A D C$ parametric response maps. PloS one. 2017;12(4):e0174620.

14. Kim JY, Park JE, Jo Y, Shim WH, Nam SJ, Kim JH, et al. Incorporating diffusion- and perfusionweighted MRI into a radiomics model improves diagnostic performance for pseudoprogression in 
glioblastoma patients. Neuro Oncol. 2019;21(3):404-14.

15. Mehta S, Gajjar SR, Padgett KR, Asher D, Stoyanova R, Ford JC, et al. Daily Tracking of Glioblastoma Resection Cavity, Cerebral Edema, and Tumor Volume with MRI-Guided Radiation Therapy. Cureus. 2018;10(3):e2346.

16. Mutic S, Dempsey JF. The ViewRay system: magnetic resonance-guided and controlled radiotherapy. Semin Radiat Oncol. 2014;24(3):196-9.

17. Bieri O, Scheffler K. Fundamentals of balanced steady state free precession MRI. J Magn Reson Imaging. 2013;38(1):2-11.

18. Chavhan GB, Babyn PS, Jankharia BG, Cheng HL, Shroff MM. Steady-state MR imaging sequences: physics, classification, and clinical applications. Radiographics : a review publication of the Radiological Society of North America, Inc. 2008;28(4):1147-60.

19. Hanicke W, Vogel HU. An analytical solution for the SSFP signal in MRI. Magnetic resonance in medicine. 2003;49(4):771-5.

20. Macdonald DR, Cascino TL, Schold SC, Jr., Cairncross JG. Response criteria for phase II studies of supratentorial malignant glioma. J Clin Oncol. 1990;8(7):1277-80.

21. Sanghera P, Perry J, Sahgal A, Symons S, Aviv R, Morrison M, et al. Pseudoprogression following chemoradiotherapy for glioblastoma multiforme. Can J Neurol Sci. 2010;37(1):36-42.

22. Galban CJ, Chenevert TL, Meyer CR, Tsien C, Lawrence TS, Hamstra DA, et al. The parametric response map is an imaging biomarker for early cancer treatment outcome. Nat Med. 2009;15(5):572-6.

23. Hamstra DA, Galban CJ, Meyer CR, Johnson TD, Sundgren PC, Tsien C, et al. Functional diffusion map as an early imaging biomarker for high-grade glioma: correlation with conventional radiologic response and overall survival. J Clin Oncol. 2008;26(20):3387-94.

24. Nelson SJ, Kadambi AK, Park I, Li Y, Crane J, Olson M, et al. Association of early changes in 1H MRSI parameters with survival for patients with newly diagnosed glioblastoma receiving a multimodality treatment regimen. Neuro Oncol. 2017;19(3):430-9.

25. Wernicke AG, Smith AW, Taube S, Mehta MP. Glioblastoma: Radiation treatment margins, how small is large enough? Pract Radiat Oncol. 2016;6(5):298-305.

26. Niyazi M, Brada M, Chalmers AJ, Combs SE, Erridge SC, Fiorentino A, et al. ESTRO-ACROP guideline "target delineation of glioblastomas". Radiother Oncol. 2016;118(1):35-42.

27. Gebhardt BJ, Dobelbower MC, Ennis WH, Bag AK, Markert JM, Fiveash JB. Patterns of failure for glioblastoma multiforme following limited-margin radiation and concurrent temozolomide. Radiat Oncol. 2014;9:130.

28. Dobelbower MC, Burnett lii OL, Nordal RA, Nabors LB, Markert JM, Hyatt MD, et al. Patterns of failure for glioblastoma multiforme following concurrent radiation and temozolomide. J Med Imaging Radiat Oncol. 2011;55(1):77-81. 
29. Paulsson AK, McMullen KP, Peiffer AM, Hinson WH, Kearns WT, Johnson AJ, et al. Limited margins using modern radiotherapy techniques does not increase marginal failure rate of glioblastoma. Am J Clin Oncol. 2014;37(2):177-81.

30. McDonald MW, Shu HK, Curran WJ, Jr., Crocker IR. Pattern of failure after limited margin radiotherapy and temozolomide for glioblastoma. Int J Radiat Oncol Biol Phys. 2011;79(1):130-6.

31. Rogers S, Merlo A, Berberat J, Bodis S. In regard McDonald et al., to Pattern of failure after limited margin radiotherapy and temozolomide for glioblastoma (Int J Radiat Oncol Biol Phys 2011;79:130136). Int J Radiat Oncol Biol Phys. 2011;81(1):316; author reply

32. Yang Y, Cao M, Sheng K, Gao Y, Chen A, Kamrava M, et al. Longitudinal diffusion MRI for treatment response assessment: Preliminary experience using an MRI-guided tri-cobalt 60 radiotherapy system. Med Phys. 2016;43(3):1369-73.

33. Zakariaei N, Chen Y, Nejad-Davarani SP, Haacke EM, Glide-Hurst CK. Rapid Multicontrast Brain Imaging at 0.35 to Facilitate Tumor Response Assessment and Functional Adaptive Radiation Therapy. J Med Phys. 2019;46(6):e517.

34. Wen PY, Chang SM, Van den Bent MJ, Vogelbaum MA, Macdonald DR, Lee EQ. Response Assessment in Neuro-Oncology Clinical Trials. J Clin Oncol. 2017;35(21):2439-49.

35. Gulani V, Calamante F, Shellock FG, Kanal E, Reeder SB, International Society for Magnetic Resonance in M. Gadolinium deposition in the brain: summary of evidence and recommendations. Lancet Neurol. 2017;16(7):564-70.

36. Choi YJ, Kim HS, Jahng GH, Kim SJ, Suh DC. Pseudoprogression in patients with glioblastoma: added value of arterial spin labeling to dynamic susceptibility contrast perfusion MR imaging. Acta Radiol. 2013;54(4):448-54.

37. Liu ZC, Yan LF, Hu YC, Sun YZ, Tian Q, Nan HY, et al. Combination of IVIM-DWI and 3D-ASL for differentiating true progression from pseudoprogression of Glioblastoma multiforme after concurrent chemoradiotherapy: study protocol of a prospective diagnostic trial. BMC Med Imaging. 2017;17(1):10.

38. Cuoco JA, Klein BJ, Busch CM, Guilliams EL, Olasunkanmi AL, Entwistle JJ. Corticosteroid-Induced Regression of Glioblastoma: A Radiographic Conundrum. Front Oncol. 2019;9:1288.

\section{Figures}




\section{A - T2 FSE}

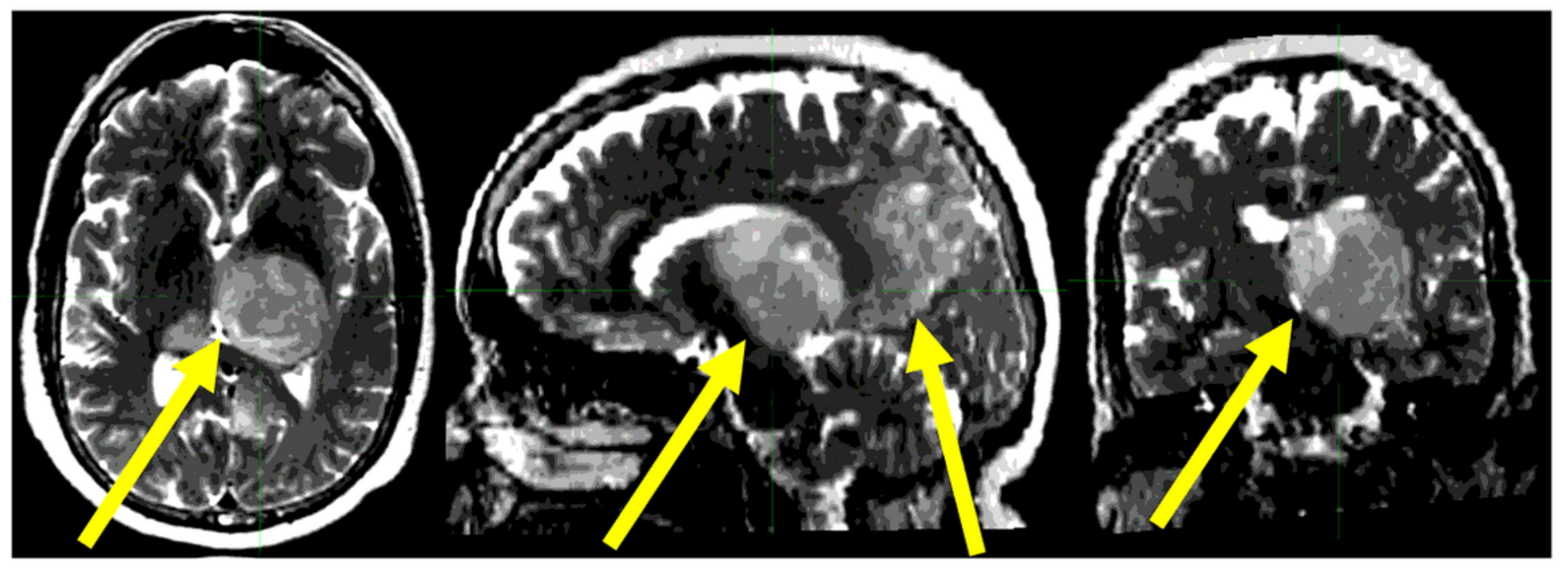

\section{$\mathrm{B}-\mathrm{bSSFP}$}

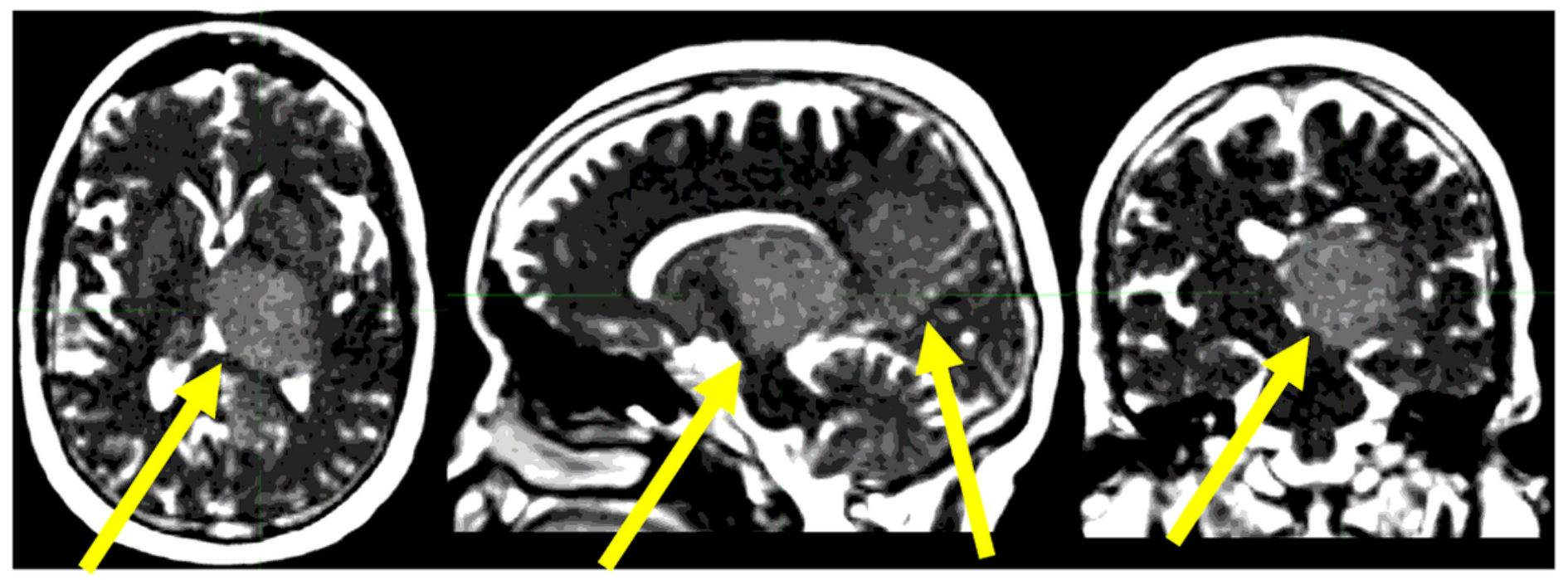

Figure 1

Brain images from patient \#7 in this study. A) Pre-treatment T2 FSE from a clinical 1.5T scanner, resolution of $0.94 \times 0.94 \times 2 \mathrm{~mm} 3$, acquisition time of 5 minutes and 43 seconds. B) bSSFP from a $0.35 T$ scanner, resolution of $1.5 \times 1.5 \times 1.5 \mathrm{~mm} 3$ (3D acquisition), acquisition time of 2 minutes and 8 seconds. Contrast and resolution are similar between the two scans. The yellow arrows indicate the MRI finding. 
A

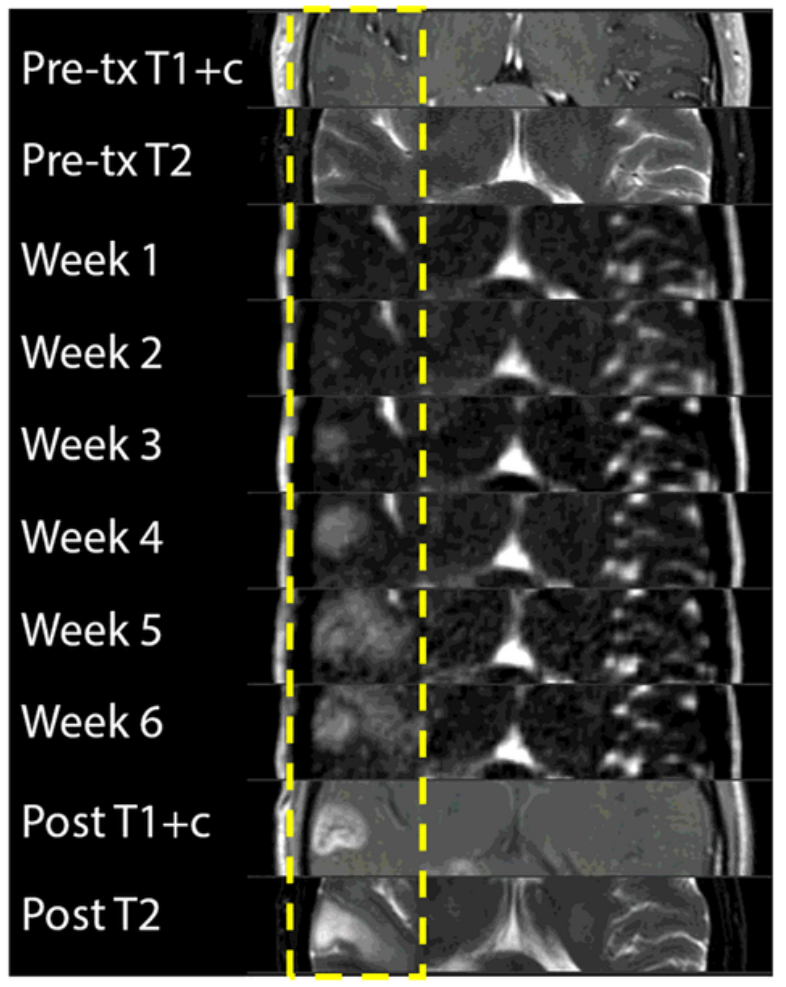

B

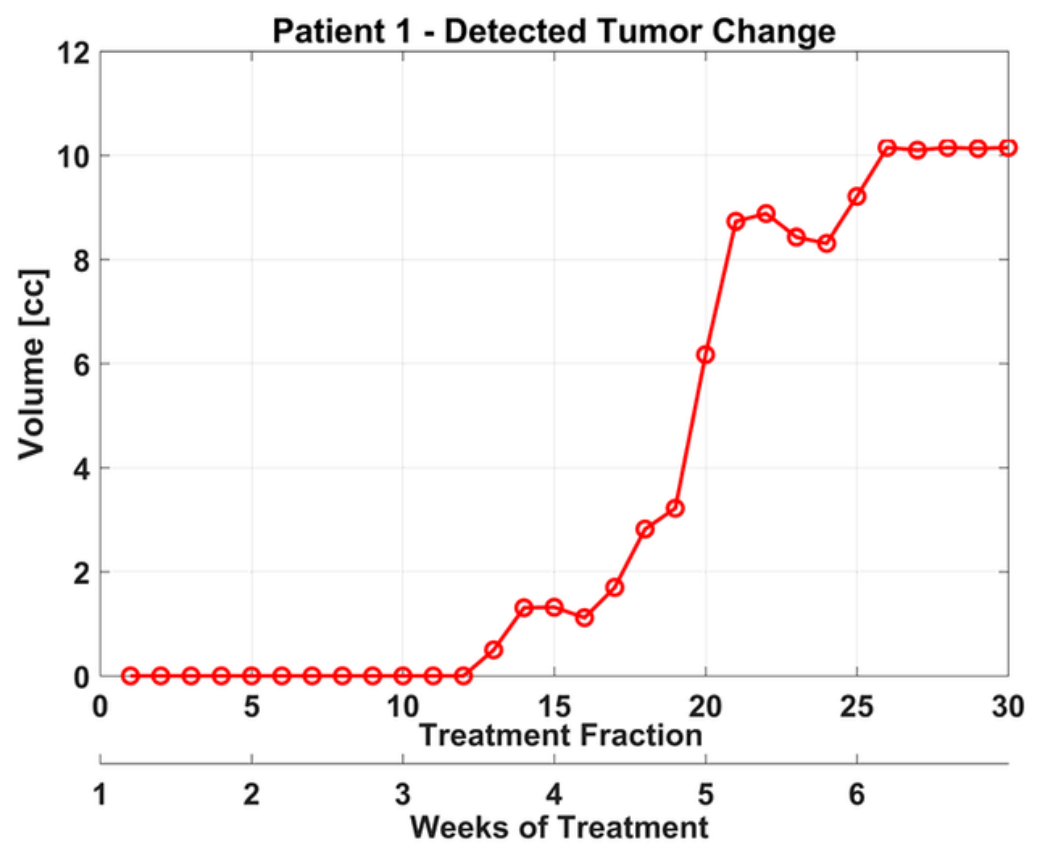

Figure 2

Volume change during treatment for patient 1. A) From top to bottom, the same axial slice of pre, intratreatment (from first to sixth week) and post MR-images. The pre-treatment (Pre-tx) and post-treatment (Post) MRIs were T1 with gadolinium $(T 1+C)$ and $T 2$ weighted. The intra-treatment images result from the mostly T2-weighted bSSFP sequence. The yellow dashed box indicates the MRI finding. B) Volume change of the MRI finding over 30 treatment fractions (six weeks). 

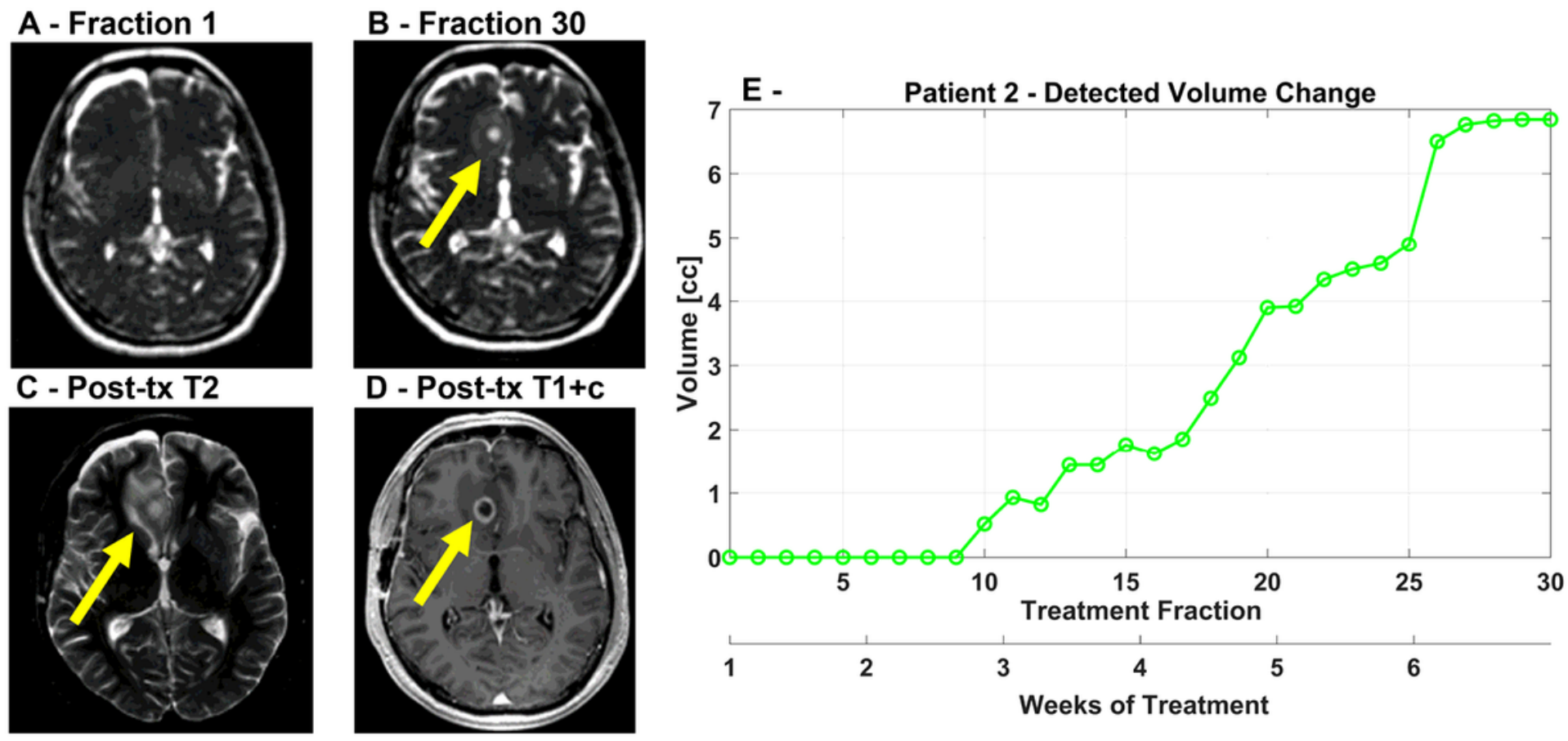

Figure 3

Volume change during treatment for patient 2. Intra-treatment axial slice of bSSFP acquisition from first (A) and thirtieth (B) treatment fractions. Post-treatment T2w (C) and T1 (D) with contrast agent weighted MRIs. The yellow arrows indicate the MRI finding. B) Volume change of the MRI finding over 30 treatment fractions (six weeks). 

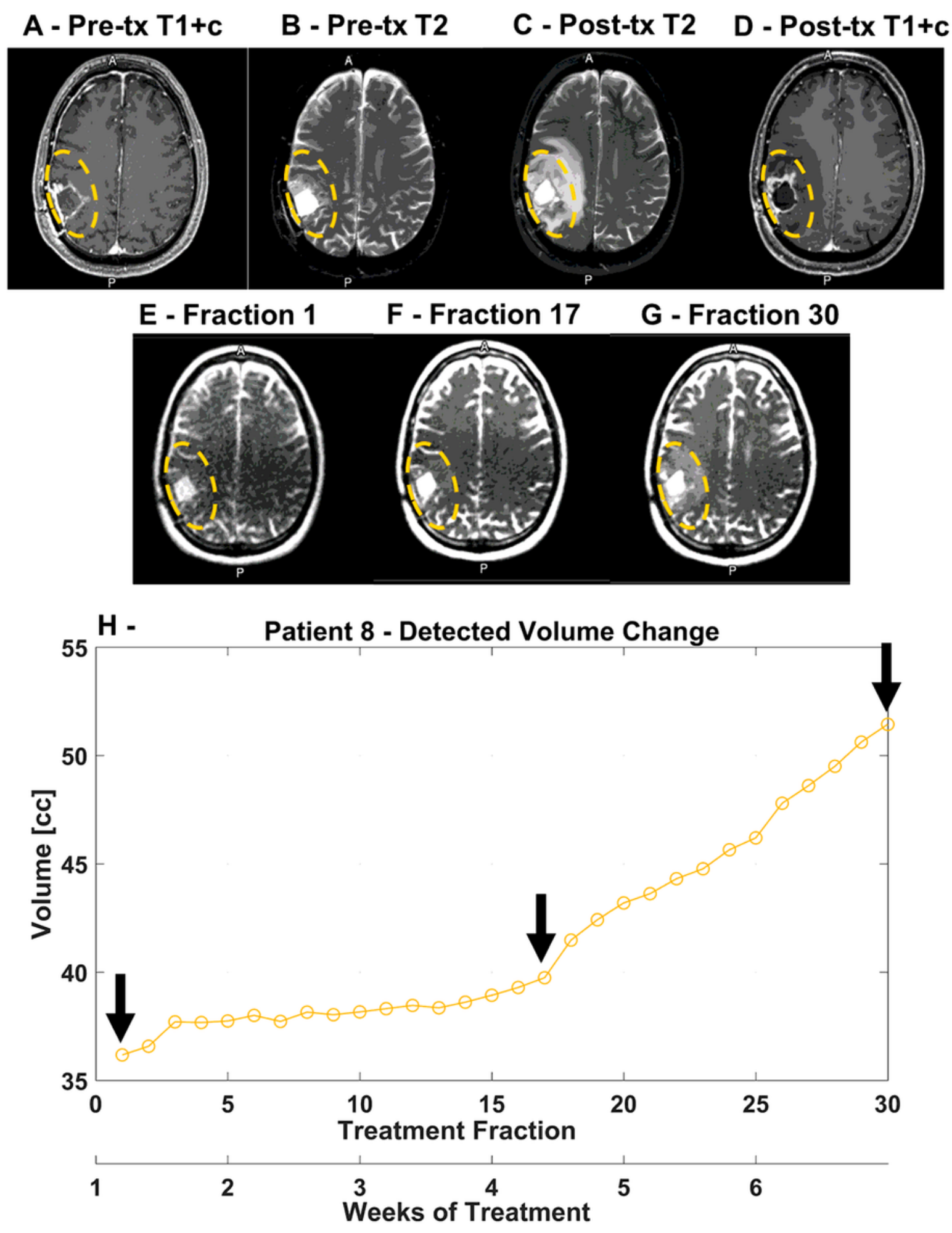

Figure 4

Volume change during treatment for patient 8 . (A) Pre-treatment diagnostic $T 1+\mathrm{C}$ scan shows gross total resection with linear likely post-operative inflammation. Pre-tx (B) and Post-tx (C) images demonstrate significant enlargement of T2-hyperintense volume, and which also correlated with $\mathrm{T} 1+\mathrm{c}$ on posttreatment MRI (D) indicating progression. Little change was observed during treatment from fraction 1 (E) to fraction $17(\mathrm{~F})$, with growth of hyperintense volume from fraction 17 to $30(\mathrm{G})$ as plotted in $(\mathrm{H})$. The 
yellow dashed ellipse line shows the maximal extent of hyperintensity at fraction 30 , with likely continued progression Post-Tx.
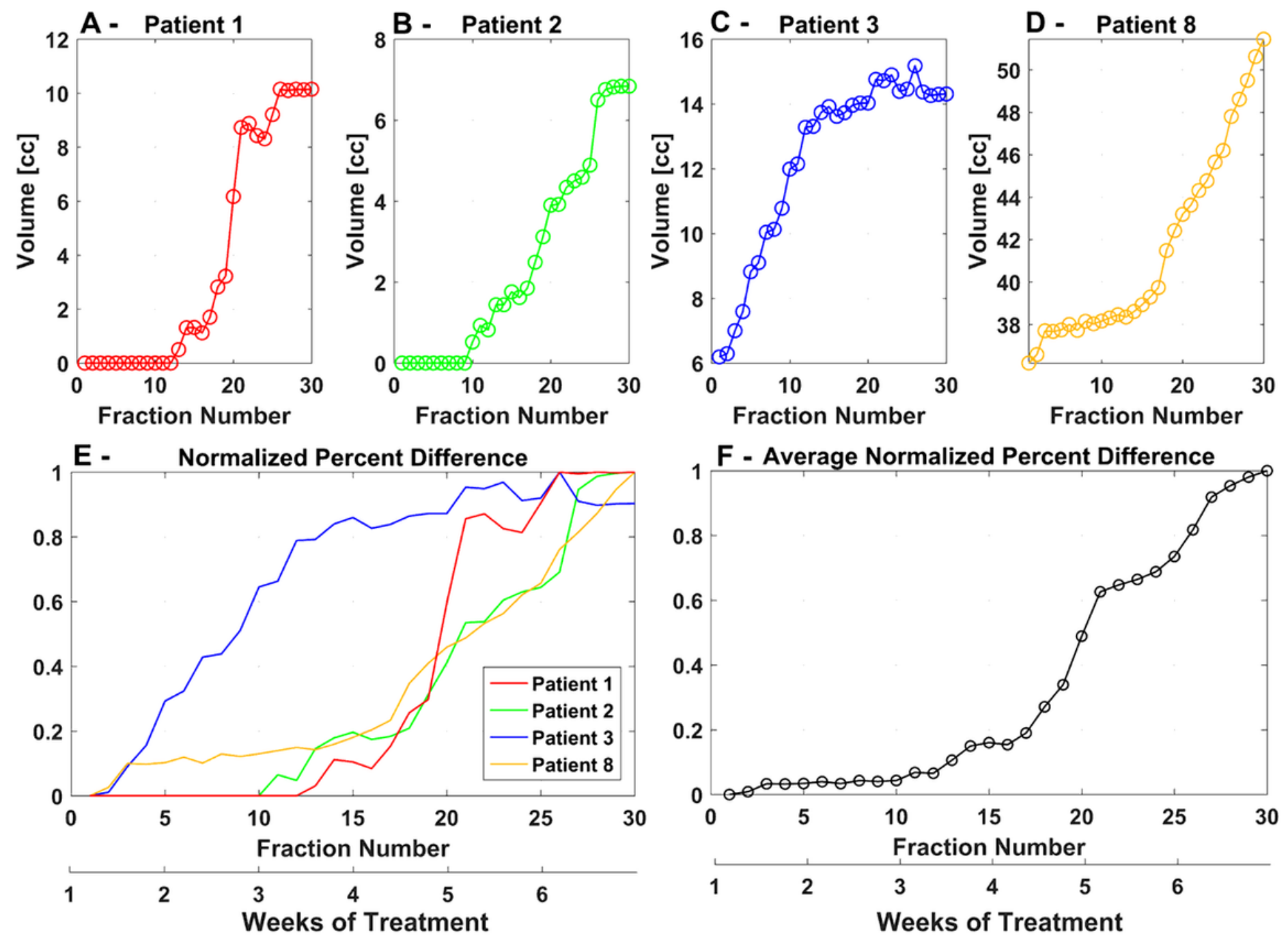

Figure 5

Volume change of the MRI finding over 30 treatment fractions for patients 1 (A), 2 (B), 3 (C) and 8 (D). E) Normalized percent difference for patients 1, 2, 3 and 8 over 30 treatment fractions (six weeks). F) Average normalized percent difference from patients 1,2, and 8 over 30 treatment fractions (six weeks). 


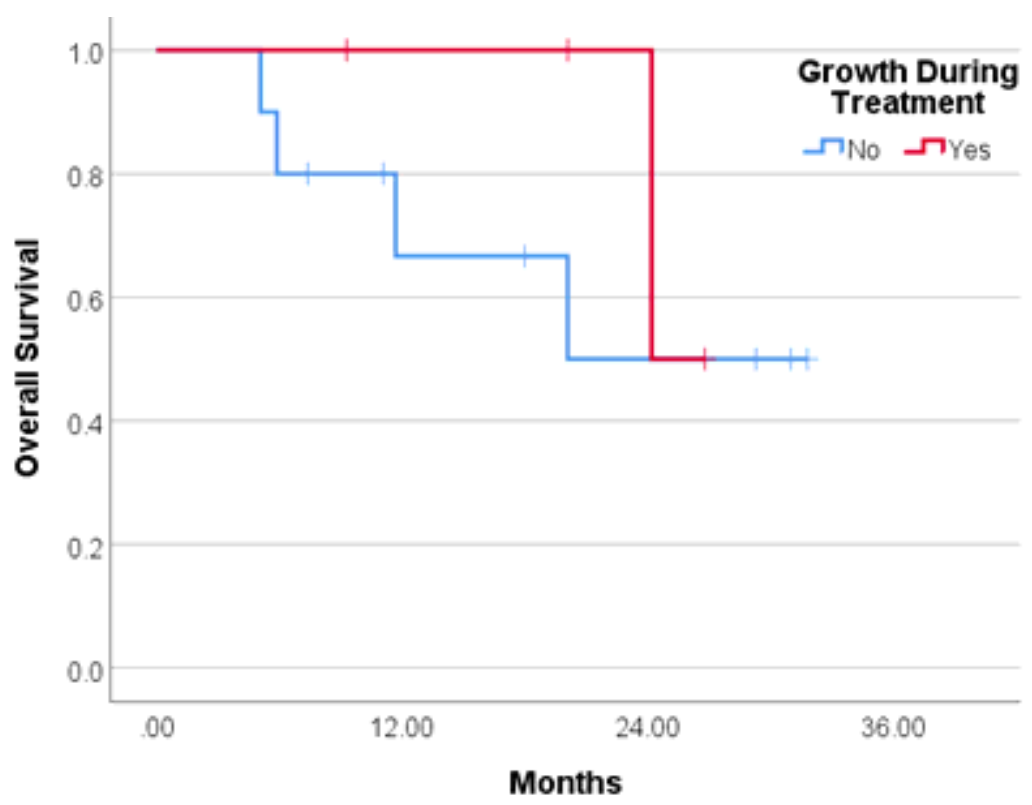

Figure 6

Kaplan-Meier estimated survival of patients with (red line) or without (blue line) $25 \%$ or more volume change during primary chemoradiotherapy. This difference was not statistically significant. 